\title{
QUIMIOPROFILAXIA DE HERPES GENITAL
}

\author{
Ana Isabel Teixeira', Nuno Vaz', João Borges da Costa ${ }^{2}$ \\ IInterno(a) do Internato Complementar de Dermatologia e Venereologia/Resident, Dermatology and Venereology \\ 2 Professor Doutor, Especialista em Dermatologia e Venereologia/Professor and Consultant, Dermatology and \\ Venereology \\ Clínica Universitária de Dermatologia de Lisboa, Hospital Santa Maria, Centro Hospitalar Lisboa Norte, Faculdade de \\ Medicina de Lisboa, Universidade de Lisboa; Instituto de Higiene e Medicina Tropical, Universidade Nova de Lisboa, \\ Portugal
}

RESUMO - O herpes genital é uma das doenças sexualmente transmissíveis mais comuns, com distribuição mundial. Os episódios recorrentes desta doença estão associados a um grau de morbilidade importante pelo que a quimioprofilaxia das recorrências assume um papel relevante na qualidade de vida dos doentes. Não existem guidelines definidas quanto aos critérios para início de terapêutica profilática, apesar de existir evidência que legitíma o início a partir das 6 recorrências anuais. Os fármacos aprovados para o efeito são o aciclovir, valaciclovir e famciclovir em diferentes esquemas e com eficácias semelhantes. Nos doentes co-infetados com os vírus herpes símplex (VHS) e vírus da imunodeficiência humana $(\mathrm{VIH})$ estes fármacos mantém-se eficazes em esquemas com doses mais elevadas. O perfil de segurança destas terapêuticas foi comprovado em estudos com tempos de seguimento até 18 anos e o aparecimento de resistências, apesar de raro na população imunocompetente (1\%), é um fator preocupante em doentes imunocomprometidos nos quais pode chegar aos 10,9\%. Várias terapêuticas alternativas têm vindo a ser estudadas no controlo desta doença, como a vacina terapêutica, novos fármacos com mecanismos de atuação diferente, mas todas permanecem em fase de investigação.

PALAVRAS-CHAVE - Herpes genital; Recorrência; Aciclovir; Valaciclovir; Famciclovir.

\section{CHEMOPROPHYLAXIS OF GENITAL HERPES}

ABSTRACT - Genital herpes is among the most common sexually transmitted diseases worldwide. Recurrent genital herpes is associated with major morbidity. Therefore, suppressive therapy plays an important role in the life quality of these patients. There are no established guidelines regarding the criteria for initiation of prophylactic therapy, although there is evidence for it after 6 recurrences per year. Drugs approved for this purpose are acyclovir, valacyclovir and famciclovir in different regimens with similar efficacy. In patients co-infected with human immunodeficiency virus (HIV) and herpes simplex virus (HSV), these drugs remain effective with higher doses regimens. The safety profile of this therapy has been proven in studies with follow-up times up to 18 years and the appearance of resistance although rare in the immunocompetent population (1\%), is a fator of concern in immunocompromised patients reaching $10.9 \%$. Several alternative therapies have been studied, such as therapeutic vaccine or new drugs with different action targets, but all remain under investigation.

KEY-WORDS - Herpes genitalis/drug therapy ; Recurrence; Acyclovir; Valaciclovir; Famciclovir.

\footnotetext{
Conflitos de interesse: Os autores declaram não possuir conflitos de interesse.

No conflicts of interest.

Suporte financeiro: $O$ presente trabalho não foi suportado por nenhum subsídio ou bolsa.

No sponsorship or scholarship granted.
}

Recebido/Received - Março/March2013; Aceite/Accepted - Abril/April 2013 


\title{
GEIDST
}

\author{
Dr. $\underline{a}$ Ana Isabel Teixeira \\ Clínica Universitária de Dermatologia de Lisboa \\ Hospital Santa Maria - Centro Hospitalar Lisboa Norte \\ Avenida Professor Egas Moniz \\ 1649-035 Lisboa, Portugal \\ Tel: +351217805000 \\ E-mail: anaisa_tx@hotmail.com
}

O Herpes Genital é uma doença sexualmente transmissível causada pelo vírus herpes simplex tipo 2 (VHS-2) em $60-80 \%$ dos casos e pelo VHS-1 em 35\% dos casos ${ }^{1}$. Estudos epidemiológicos estimaram uma seroprevalência do VHS-2 de $15-25 \%$ na população geral dos países desenvolvidos, mas este valor pode chegar aos $80-90 \%$ noutros grupos, como indivíduos infetados pelo $\mathrm{VIH}$ ou que se dedicam à prostituição ${ }^{2}$.

Trata-se de uma infeção crónica que não pode ser erradicada, caracterizada por episódios recorrentes após a primoinfeção. As recorrências acontecem em $20-50 \%$ dos doentes infetados, numa média de 4-5 episódios no primeiro ano após o primeiro evento sintomático e tendem a tornar-se menos frequentes a partir daí. O VHS-1 é responsável por 4 vezes menos recorrências que o $\mathrm{VHS}-2^{3}$.

Durante os períodos assintomáticos ocorre libertação episódica do vírus através da mucosa genital (cervix, vulva e zona perianal na mulher e pele peniana, uretra e região perianal no homem). Esta libertação é mais frequente no VHS-2 quando comparada com o VHS- 1 e está associada a um número superior de recorrências, aquisição recente da infeção ou estados de co-infeção com o vírus da imunodeficiência humana $(\mathrm{VIH})^{4}$. Vários estudos concluíram que a transmissão viral ocorre frequentemente durante estes períodos de libertação assintomática e um indivíduo infetado pode transmitir o vírus independentemente da presença lesões ${ }^{5}$.

Os episódios recorrentes de herpes genital estão associados a um grau de morbilidade elevado, stress psicológico e estigmatização social, com uma consequente diminuição da qualidade de vida ${ }^{6}$. A sua abordagem passa pelo tratamento episódico das recorrências ou tratamento supressivo (contínuo) com - objetivo de limitar a reativação viral. A profilaxia tem uma eficácia comprovada de $70-80 \%$ na diminuição das recorrências, além de diminuir a libertação viral assintomática pela mucosa genital ${ }^{7}$, contribuindo para uma redução de aproximadamente $50 \%$ da transmissão do vírus em casais heterossexuais serologicamente discordantes ${ }^{8}$.

A escolha de iniciar terapêutica profilática vs terapêutica episódica é subjetiva e é influenciada pelo número de recorrências e sua gravidade, pelo estado serológico do parceiro, estados de imunossupressão, e por outros fatores como impacto psicossocial da doença e relação custo-benefício ${ }^{6}$. A maioria dos estudos realizados sobre este tema recomenda iniciar tratamento profilático em doentes com 6 ou mais recorrências anuais, ou apenas 4 se classificadas como graves 9 . Em casais serodiscordantes e grupos de risco, como homens que têm sexo com homens e prostitutas, pode ser instituída esta terapêutica com o intuito de diminuir o risco de transmissão ${ }^{10}$.

Apesar de não existirem guidelines definidas quanto à duração da terapêutica supressiva, está demonstrado que ao final de um ano há diminuição das taxas de recorrência para níveis inferiores aos basais, pelo que, periodicamente (12-24 meses) se deve suspender o tratamento para avaliar o perfil de recidiva a partir daí ${ }^{10}$. O período de avaliação deve incluir pelo menos duas recorrências e os pacientes que mantenham taxas de recorrência elevadas devem reiniciar a quimioprofilaxia"1.

Os fármacos aprovados para este fim são o aciclovir, valaciclovir e famciclovir. Os vários estudos efetuados até agora demonstraram um ótimo perfil de segurança com um tempo máximo de seguimento de 18 anos para o aciclovir e 1 ano para o valaciclovir e famciclovir ${ }^{12}$.

Os esquemas terapêuticos recomendados atualmente em doentes imunocompetentes estão resumidos na Tabela 1.

A eficácia dos diferentes esquemas é semelhante com a exceção do grupo de doentes com doença recorrente superior a 10 recidivas anuais em que foi provada um vantagem dos esquemas de aciclovir ou valaciclovir $1 \mathrm{~g}$ per os id sobre valaciclovir $500 \mathrm{mg}$ per os id. ${ }^{13}$. Todos estes fármacos promovem uma 
Tabela 1 - Esquemas terapêuticos recomendados para quimioprofilaxia de herpes genital em doentes imunocompetentes

\begin{tabular}{lc}
\hline Aciclovir & $400 \mathrm{mg}$ per os bid \\
\hline Famciclovir & $250 \mathrm{mg}$ per os bid \\
\hline Valaciclovir & $500 \mathrm{mg}$ per os id \\
\hline Valaciclovir & $1 \mathrm{~g}$ per os id \\
\hline Adaptado de Center of diseases control and prevention, Sexually Transmitted Diseases Treatment
\end{tabular}

Adaptado de Center of diseases control and prevention, Sexually Transmitted Diseases Treatment Guidelines, 2010.

diminuição da libertação assintomática do vírus mas o famciclovir parece ser menos eficaz comparativamente ao valaciclovir ${ }^{13}$.

Em doentes co-infetados com o VHS e VIH as recorrências de herpes genital tendem a ser mais frequentes e mais graves ${ }^{14}$. Estudos epidemiológicos sugerem que a infeção por VHS é fator promotor da infeção por $\mathrm{VIH}$, não só pela disrupção da mucosa anogenital que condiciona, mas também pelo aumento da libertação de $\mathrm{VIH}$ na mucosa genital, mesmo em doentes sem úlcera ativa. Sabe-se que a quimioprofilaxia do VHS diminui a carga viral detetável (RNA) de VIH-1 quer no plasma quer na mucosa ano-genital ${ }^{15}$, e que a terapêutica anti-retrovírica tem um papel ativo na diminuição da taxa de recorrências de herpes genital.

Os esquemas terapêuticos recomendados atualmente em doentes infetados com $\mathrm{VIH}$ - 1 estão resumidos na Tabela 2.

Tabela 2 - Esquemas terapêuticos recomendados para quimioprofilaxia de herpes genital em doentes imunocomprometidos

\begin{tabular}{|l|c}
\hline Aciclovir & $400-800 \mathrm{mg}$ per os bid-tid \\
\hline Famciclovir & $500 \mathrm{mg}$ per os bid \\
\hline Valaciclovir & $500 \mathrm{mg}$ per os bid \\
\hline
\end{tabular}

Adaptado de Center of diseases control and prevention, Sexually Transmitted Diseases Treatment Guidelines, 2010.

Outros indivíduos com imunossupressão, como doentes com alterações hematológicas, transplantados medulares ou sob terapêutica imunossupressora, obedecem aos mesmos esquemas de profilaxia delineados para os doentes com VIH. No entanto, doentes sob terapêutica profilática anti-vírica com ganciclovir ou valganciclovir para o citomegalovírus encontram-se já protegidos contra reativações de herpes genital ${ }^{1}$.

A quimioprofilaxia de recorrências de herpes genital em mulheres grávidas não está indicada, passando a sua orientação apenas pela prevenção da transmissão fetal durante o parto. Sabe-se que o risco de transmissão é elevado (30-50\%) entre grávidas que adquirem a infeção durante 0 terceiro trimestre, mas baixo (1\%) quando a infeção é adquirida previamente ou no princípio da gravidez. No início do trabalho de parto todas as mulheres devem ser avaliadas quanto à presença de lesões herpéticas ou pródromos. Se estes estiverem ausentes o risco de transmissão é desprezível, podendo o parto ocorrer por via vaginal. $O$ contrário implica a realização de cesariana ${ }^{9}$.

O aciclovir pode ser administrado na gravidez apenas em casos de primoinfeção ou episódio recorrente grave. A segurança da administração sistémica de aciclovir, valaciclovir e famciclovir em grávidas não se encontra comprovada ${ }^{10}$. Contudo, não estão descritos, até à data, riscos acrescidos de malformações fetais com o uso de aciclovir no primeiro trimestre de gravidez, estando por isso, inserido na categoria de risco $C$ de acordo com os critérios da Food and Drug Administration. A informação disponível sobre o valaciclovir e o famciclovir é muito escassa, pelo que não está definido o seu nível de risco.

Quando não existe resposta clínica satisfatória com utilização de esquemas recomendados, a hipótese de resistência ao fármaco utilizado deve ser considerada e comprovada com testes de senibilidade in vitro sempre que possível. A taxa de resistência ao aciclovir em doentes imunocompetentes é inferior a $1 \%$ e tem-se mantido estável nos últimos 20 anos $^{16}$. Pelo contrário, em doentes infetados com $\mathrm{VIH}$, este valor pode subir aos 3,6-10,9\% ${ }^{17}$. Todas as estirpes resistentes ao aciclovir são resistentes ao valaciclovir e a maioria é também resistente ao famciclovir. As opções terapêuticas passam pela utilização de antivíricos com diferentes mecanismos de ação como foscarnet ou cidofovir em esquemas que ainda não estão definidos para este fim $^{18}$. Estes doentes devem ser também referenciados a especialistas em $\mathrm{VIH}$.

O desenvolvimento de vacinas terapêuticas que modulam a resposta imunitária poderá ser o próximo passo na profilaxia das recorrências de herpes genital, contudo são necessários mais estudos para comprovar a sua eficácia ${ }^{19}$. Os antagonistas dos toll like receptors como o imiquimod e resiquimod têm sido avaliados quanto à sua eficácia contra o VHS, e poderá vir a ser explorado o seu potencial no tratamento, supressão e prevenção das infeções por $\mathrm{VHS}^{20}$.

Em 2013, o controlo das recorrências de herpes genital permanece um objetivo atual pelo impacto que esta doença tem na qualidade de vida de milhões de 
pessoas e pela sua relação com a transmissão de outras doenças sexualmente transmissíveis, nomeadamente o $\mathrm{VIH}$.

\section{BIBLIOGRAFIA}

1. Kimberlin DW, Rouse DJ. Genital herpes. N Engl J Med. 2004; 350:1970-7.

2. Whitley RJ, Roizman B. Herpes simplex virus infections. Lancet. 2001; 357:1513-8.

3. Benedetti J, Corey L, Ashley R. Recurrence rates in genital herpes after symptomatic first-episode infection. Ann Intern Med. 1994; 121:847-54.

4. Tronstein E, Johnston C, Huang ML, Selke S. Genital Shedding of Herpes Simplex Virus Among Symptomatic and Asymptomatic Persons With HSV-2 Infection. JAMA. 2011; 305(14):1441-9.

5. Patel R, Bodsworth NJ, Woolley P, Peters B, Vejlsgaard G, Saari $S$, et al. Valaciclovir for the suppression of recurrent genital HSV infection: a placebo controlled study of once daily therapy. International Valaciclovir HSV Study Group. Genitourin Med. 1997; 73:105-9.

6. Scalone L, Watson V, Ryan M, Kotsopoulos N, Patel $R$. Evaluation of patients' preferences for genital herpes treatment. Sex Transm Dis. 201 1; 38(9):802-7.

7. Wald A, Zeh J, Barnum G, Davis LG, Corey L. Suppression of subclinical shedding of herpes simplex virus type 2 with acyclovir. Ann Intern Med. 1996; 124:8-15.

8. Corey L, Wald A, Patel R, Sacks SL, Tyring SK, Warren $T$, et al. Once-daily valacyclovir to reduce the risk of transmission of genital herpes. N Engl J Med. 2004; 350(1):11-20.

9. Patel R, Alderson S, Geretti A, Nilsen A, Foley E, Lautenschlager $S$, et al, European guideline for the management of genital herpes, 2010. Int J STD AIDS. 2011; 22(1):1-10.

10. Centers for Disease Control and Prevention. Sexually Transmitted Diseases Treatment Guidelines,
MMWR 2010; 59.

11. Clinical Effectiveness Group (British Association for Sexual Health and HIV) National Guideline for the Management of Genital Herpes 2007. Cheshire: BASH; 2007. [consultado em Dez 2012]. Disponível em: http://www.bashh.org/guidelines

12. Cernik C, Gallina K, Brodell RT. The treatment of herpes simplex infections: an evidence-based review. Arch Intern Med. 2008; 168(1 1):1137-44.

13. Lebrun-Vignes B, Bouzamondo A, Dupuy A, Guillaume JC, Lechat $P$, Chosidow O. A meta-analysis to assess the efficacy of oral antiviral treatment to prevent genital herpes outbreaks. J Am Acad Dermatol. 2007; 57:238-46.

14. Freeman EE, Weiss HA, Glynn JR, Cross PL, Whitworth JA, Hayes RJ. Herpes simplex virus 2 infection increases HIV acquisition in men and women: systematic review and meta-analysis of longitudinal studies. AIDS. 2006;20(1):73-83

15. Nagot N, Ouedraogo A, Foulongne V, Konaté I, Weiss HA, Vergne L, et al. Reduction of HIV-1 RNA levels with therapy to suppress herpes simplex virus. N Engl J Med. 2007;356(8):790-9.

16. Reyes M, Shaik NS, Graber JM, Nisenbaum R, Wetherall NT, Fukuda K, et al. Acyclovir-resistant genital herpes among persons attending sexually transmitted disease and human immunodeficiency virus clinics. Arch Intern Med. 2003;163(1):76-80.

17. Levin MJ, Bacon TH, Leary JJ. Resistance of herpes simplex virus infections to nucleoside analogues in HIV-infected patients. Clin Infect Dis. 2004; 39(Suppl 5):S248-S257.

18. Wagstaff AJ, Bryson HM. Foscarnet. A reappraisal of its antiviral activity, mviral infections. Drugs .1994; 48: 199-226.

19. Wilson SS, Fakioglu E, Herold BC. Novel approaches in fighting herpes simplex virus infections. Expert Rev Anti Infect Ther. 2009;7(5):559-68.

20. Strick LB, Wald A, Celum C. Management of herpes simplex virus type 2 infection in HIV type 1-infected persons. Clin Infect Dis. 2006; 43:347-56. 